\title{
Antenatal Diagnosis of Corpus Callosal Agenesis
}

\author{
Amir Irfan Kazi ${ }^{1,2}$ (1) $\cdot$ Yasmin Iqbal $^{2,3} \cdot$ Sana Elham Kazi ${ }^{2}$
}

Received: 1 February 2015/ Accepted: 5 June 2015/Published online: 17 June 2015

(C) Society of Fetal Medicine 2015

\begin{abstract}
The corpus callosum (CC) is the largest interhemispheric commissure connecting the neocortical areas of the two cerebral hemispheres. The spectrum of anomalies of the $\mathrm{CC}$ includes agenesis, which may be complete or partial, and hypoplasia. The callosal agenesis may be isolated or may be associated with other abnormalities. Nonvisualization of cavum septum pellucidum and colpocephaly are important signs in the axial view which aid in the diagnosis. Demonstrating the absence of $\mathrm{CC}$ in midsagittal plane is the gold standard. We report a case of isolated complete corpus callosal agenesis diagnosed antenatally in the third trimester with a brief review of ultrasound findings in corpus callosal agenesis.
\end{abstract}

Keywords Corpus callosal agenesis · Ventriculomegaly · Nonvisualization of cavum septum pellucidum .

Colpocephaly

\section{Introduction}

The corpus callosum (CC) is the largest interhemispheric commissure, consisting of over 200 million nerve fibers connecting the neocortical areas of the two cerebral hemispheres [1]. It plays a major role in the integration of

Amir Irfan Kazi

kaziamirirfan@gmail.com

1 Department of Radiology, The Muslim Maternity and General Hospital, Hyderabad, India

2 Centre for Obstetrics, Gynaecology and Infertility, New Malakpet, 16-8-646, Hyderabad 500024, Telangana, India

3 Department of Obstetrics and Gynaecology, The Muslim Maternity and General Hospital, Hyderabad, India the cerebral functions [2]. CC is sub-divided into a central body, posterior splenium, and anterior genu which folds on itself and ends in the reflected anterior part, the rostrum. During development, the posterior portion of genu appears first, followed by the anterior body. The anterior portion of genu and the posterior body develop next, around the same time followed by the splenium. The rostrum is the last to appear [3].

The spectrum of anomalies of the CC includes agenesis, which may be complete or partial and hypoplasia. Partial agenesis may be due to incomplete formation (hypogenesis), defective formation (dysgenesis), and destruction [4, 5]. Hypogenesis is due to abnormality in development. In defective formation, the parts which form later during development are seen, while those which form earlier, may be absent. It is seen in conditions like classic holoprosencephaly where splenium may be present with absence of genu and body. Destruction of portions of CC due to antenatal insult may also result in complete or partial agenesis [4]. The callosal agenesis may be isolated or may be associated with other abnormalities.

\section{Report of Case}

A 29-year-old third gravida, with nonconsanguineous marriage, came to our ultrasound department for third trimester scan, referred to us for evaluation of ventriculomegaly. The gestational age of the fetus was 33 weeks by her last menstrual period. On ultrasound examination, increased head circumference was seen, which was within 95th percentile range. Polyhydramnios was seen. On evaluation of the fetal brain, ventriculomegaly was seen with the occipital horns of bilateral lateral ventricles dilated with pinched and widely-separated frontal horns 
showing "tear drop" configuration (Fig. 1a). The cavum septum pellucidum (CSP) was not visualized (Fig. 1b). These features were suggestive of $\mathrm{CC}$ agenesis. The third ventricle appeared elevated. Fourth ventricle was within normal limits. The posterior cranial fossa was unremarkable. Detailed evaluation of the fetus revealed no other associated congenital anomaly. However, there was associated single umbilical artery. The baby was delivered by elective cesarean. On clinical examination, the baby showed no dysmorphic features. The fetal neurological examination was normal. The neonatal neurosonogram findings were consistent with the antenatal diagnosis. The magnetic resonance imaging (MRI) done in the neonate did not reveal any additional finding.

\section{Discussion}

The spectrum of anomalies of the $\mathrm{CC}$ includes agenesis, which may be complete or partial, and hypoplasia. The prevalence in general population ranges from 1.8 to 7 per 10,000 births and may be seen in up to $2 \%-3 \%$ neuropediatric patients [6]. It may be sporadic or a genetic mutation may be present. Chromosomal abnormalities including trisomy $13,18,8$ and triploidy may be present. It may also be seen as a part of multiple syndromes. Infections (cytomegalovirus, rubella), teratogens (alcohol, cocaine), infarct, and bleed have also been implicated as causative agents [7]. The most common associated central nervous system (CNS) anomalies include abnormalities of the other telencephalic commissures (anterior commissure and the hippocampal commissure), Dandy-Walker spectrum, cerebellar hypoplasia, Chiari II malformation, migration abnormalities, heterotopias, polymicrogyria, and encephalocele [8].
The CC can be evaluated by the two dimensional (2D) and three dimensional (3D) techniques. In 2D, the $\mathrm{CC}$ can be directly visualized in the coronal and mid-sagittal planes. Demonstrating the $\mathrm{CC}$ in the mid-sagittal plane is ideal because it can be visualized in its entirety. The key to demonstration is to utilize the acoustic window available in the metopic suture, the anterior fontanelle, and the sagittal suture. Good-quality 2D grayscale imaging is generally adequate for evaluation of the $\mathrm{CC}$ [9]. In 3D, two techniques can be used for demonstration of $\mathrm{CC}-$ (a) acquisition in the mid-sagittal plane using the acoustic window as described previously, (b) by acquiring the volume in the axial plane and then reconstructing using multiplanar imaging or volume contrast imaging [10].

Corpus callosal agenesis is prenatally diagnosed by the absence of $\mathrm{CC}$ in the mid-sagittal plane. Direct imaging the $\mathrm{CC}$ is not a part of the basic evaluation of the CNS, done in the routine anomaly scan as only axial views are recommended in the basic CNS evaluation [11]. The abnormality of $\mathrm{CC}$ is inferred based on the indirect markers, which when present, arouse the suspicion of callosal abnormality. The indirect cerebral findings seen in the transventricular plane of the axial scan include nonvisualization of the cavum septum pellucidum and ventriculomegaly. Widening of the interhemispheric fissure may also be seen [5].

The CSP is always visualized at 18-37 weeks of gestation [11]. Nonvisualization of the CSP may be either due to agenesis, or lack of formation or obliteration of space between the laminae septi pellucidi [12]. It is a marker for agenesis of $\mathrm{CC}$, but not specific for this condition. It may also not be visualized in holoprosencephaly, septo-optic dysplasia, hydrocephalus, schizencephaly, porencephaly, and hydranencephaly [4]. Nonvisualization may sometimes be an isolated finding without any associated abnormality [12]. The CSP should always be evaluated in
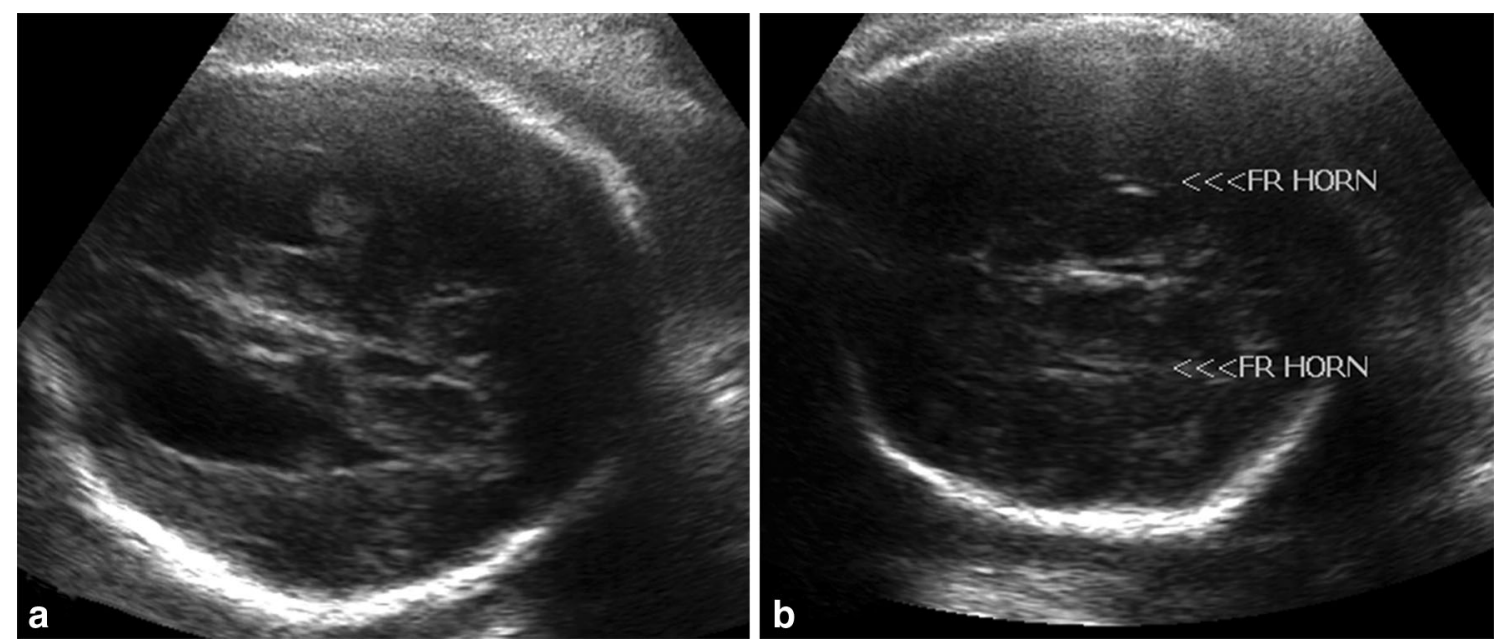

Fig. 1 a Axial view showing dilated occipital horn (colpocephaly) with narrowing of the frontal horn giving rise to 'tear drop'configuration, b coronal view showing nonvisualization of cavum septum pellucidum and widely placed frontal horns 
conjunction with the two frontal horns which lie on either side of it. In its absence, high-riding third ventricle, fused frontal horns, and small interhemispheric cysts may be mistaken for the CSP [13]. The relationship between the CSP and the frontal horns is better depicted in the coronal plane [7].

The abnormalities of the configuration of ventricular system are secondary to a lack of moulding effect by the CC. The findings include lateral displacement with parallel arrangement of the medial walls of the lateral ventricles. This is due to the Probst bundles which run along their medial walls. The occipital horns show expansion (colpocephaly) due to the absence of the posterior portion of the CC [5]. The frontal horns are widely separated and show concave medial borders with pinched appearance showing a 'viking helmet' sign due to bulge in their medial aspect by the Probst bundle. The frontal horns are prevented from expanding laterally by the caudate and lentiform nuclei. As a result of these anatomical changes, the lateral ventricle assumes a 'tear drop' configuration in the axial view. The tear drop configuration is specific for agenesis of CC. The third ventricle appears elevated reaching the level of lateral ventricles [7]. Widening of interhemispheric fissure is due to increased separation of the cerebral hemispheres. In the upper portion of the cranium, the mid-line falx and the medial borders of the two cerebral hemispheres give rise to three parallel echogenic lines [5, 14].

Demonstrating the absence of CC in mid-sagittal plane is the gold standard for diagnosis and helps in differentiating between complete and partial agenesis [9]. In the absence of $\mathrm{CC}$, there is abnormality in the gyral pattern of the medial surface of the cerebral hemispheres with absence of the cingulate gyrus and radial arrangement of the sulci which converge towards the third ventricle (Fig. 2a, b). On Doppler evaluation, the pericallosal artery which normally arches over the $\mathrm{CC}$, shows an abnormal course and its branches ascend linearly [5].

Associated CNS abnormalities may be present. Interhemispheric cysts may be seen, communicating (type I) and not communicating (type II) with the ventricular system. Other associated abnormalities may be diagnosed on ultrasound including posterior fossa abnormalities and Chiari II malformation. Periventricular nodular heterotopias and sulcation abnormalities may also be identified [5].

In partial agenesis, the sagittal plane shows partial formation of the CC. The pericallosal artery arches over the partially formed CC and then branches out linearly. The CSP is present in many of the cases. The callosal abnormality may be suspected due to colpocephaly with the sagittal plane showing partial formation. Sometimes, indirect signs on axial plane may be absent and the condition is diagnosed during evaluation of sagittal plane for other associated CNS anomaly [15].

The indirect markers of CC agenesis (colpocephaly and nonvisualization of CSP) become more evident after 24 weeks of gestation. Therefore, agenesis of CC may be missed on routine mid-trimester anomaly scan. Paladini et al. reported that partial agenesis of CC showed no abnormality of the transventricular view before 24 gestational weeks in $33.3 \%$ of cases [13]. The medico-legal implications of such findings are important and should be considered. Fetal MRI has a role in cases where the diagnosis is uncertain and in identifying other associated CNS anomalies, which is important, as this increases the possibility of neurological impairment in the surviving fetus [5, 6].
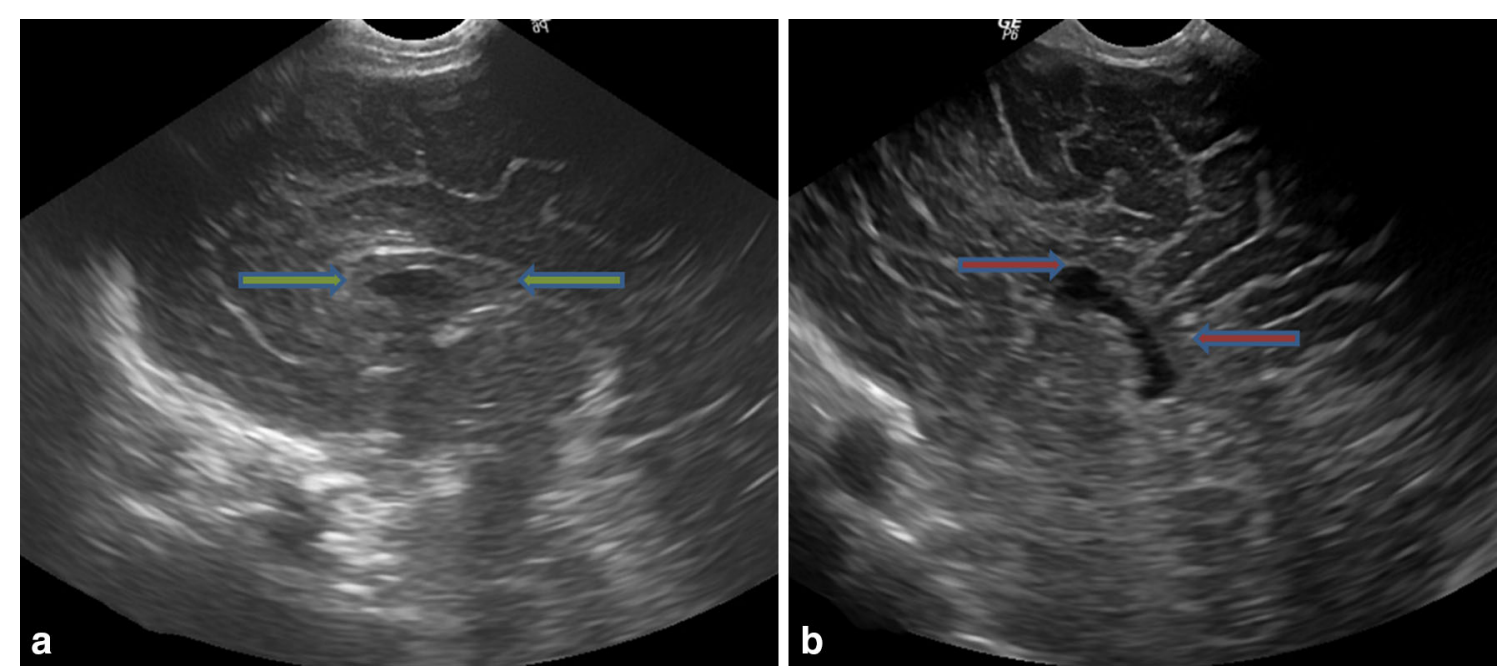

Fig. 2 a Corpus callosum (green arrows) is seen with cingulate gyrus overlying it and normal gyral arrangement, b nonvisualization of corpus callosum (red arrows) and radial arrangement of gyri 
The neurodevelopmental outcome in a fetus with $\mathrm{CC}$ agenesis depends on whether the agenesis is isolated or, associated neurological or non-neurological abnormalities are present on prenatal ultrasound, MRI, and karyotyping. When there are other associated abnormalities, the neurodevelopmental outcome is poor [5, 6]. The risk of neurodevelopmental delay in prenatally diagnosed isolated CC agenesis is about $30 \%$ [6]. The risk of neurodevelopmental delay in fetuses with partial agenesis is similar to that of the fetuses with complete agenesis [5, 15]. Postnatal work up is important as about $15 \%$ of cases thought to be isolated prenatally are found to have associated abnormalities after birth $[5,6]$.

\section{Conclusion}

Agenesis of CC may be complete or partial. Ultrasound plays an important role in diagnosis. Nonvisualization of CSP and colpocephaly are important signs in the axial view which aid in the diagnosis. Demonstrating the absence of $\mathrm{CC}$ in mid-sagittal plane is the gold standard. It may be isolated or associated with other anomalies, which can be diagnosed on ultrasound. Fetal MRI has an important role, as it helps in diagnosing other associated CNS abnormalities better.

\section{Conflict of interest None.}

Funding None.

\section{References}

1. Luders E, Thompson PM, Toga AW. The development of the corpus callosum in the healthy human brain. J Neurosci. 2010;30(33):10985-90. doi:10.1523/JNEUROSCI.5122-09.2010.
2. Gazzaniga MS. Cerebral specialization and interhemispheric communication. Brain. 2000;123(Pt 7):1293-326.

3. Losser JD, Alvord EC. Agenesis of the corpus callosum. Brain. 1968;91:553-70.

4. Barkovich AJ. Congenital malformations of the brain and skull. In: Barkovich AJ, editor. Pediatric neuroimaging. 4th ed. Philadelphia: Lippincott Williams \& Wilkins; 2005.

5. Santo S, D'Antonio F, Homfray T, et al. Counseling in fetal medicine: agenesis of the corpus callosum. Ultrasound Obstet Gynecol. 2012;40:513-21.

6. Mangione R, Fries N, Godard P, et al. Neurodevelopmental outcome following prenatal diagnosis of an isolated anomaly of the corpus callosum. Ultrasound Obstet Gynecol. 2011;37:290-5.

7. Woodward PJ. Agenesis of corpus callosum. In: Woodward PJ, Kennedy A, Sohaey R, et al., editors. Diagnostic imaging obstetrics. 1st ed. Salt Lake City: Amirsys; 2005.

8. Salomon LJ, Alfirevic Z, Berghella V, et al. Practice guidelines for performance of the routine mid-trimester fetal ultrasound scan. Ultrasound Obstet Gynecol. 2011;37(1):116-26.

9. Youssef A, Ghi T, Pilu G. How to image the fetal corpus callosum. Ultrasound Obstet Gynecol. 2013;42:718-20.

10. Pashaj S, Merz E, Wellek S. Biometry of the fetal corpus callosum by three-dimensional ultrasound. Ultrasound Obstet Gynecol. 2013;42:691-8.

11. The International Society of Ultrasound in Obstetrics and Gynaecology. Sonographic examination of the fetal central nervous system: guidelines for performing the 'basic examination' and the 'fetal neurosonogram'. Ultrasound Obstet Gynecol. 2007;29(1):109-16.

12. Malinger G, Lev D, Oren M, et al. Non-visualization of cavum septi pellucidi is not synonymous with corpus callosum agenesis. Ultrasound Obstet Gynecol. 2012;40:165-70.

13. Paladini D, Pastore G, Cavallaro A, et al. Agenesis of fetal corpus callosum: sonographic signs change with advancing gestational age. Ultrasound Obstet Gynecol. 2013;42:687-90.

14. Pilu G, Sandri F, Perolo A, et al. Sonography of fetal agenesis of the corpus callosum: a survey of 35 cases. Ultrasound Obstet Gynecol. 1993;3:318-29.

15. Ghi T, Carletti A, Contro E, et al. Prenatal diagnosis and outcome of partial agenesis and hypoplasia of the corpus callosum. Ultrasound Obstet Gynecol. 2010;35:35-41. 